\title{
Numerical Modeling of a Split Hopkinson Pressure Bar Test
}

\author{
Ahmad R. Aljohani, Ramzi Othman, and Khalid H. Almitani
}

\begin{abstract}
In this work, a one-dimensional simplified model was developed to predict stress, strain, and strain-rate in high strain rates Hopkinson pressure bar experiments, namely, between 500-5000/s. To this goal, a one-dimensional model for Hokinson bar tests was developed based on analyses of wave propagation in bars and assuming the specimen is under equilibrium during the test. The numerical tool implemented using Matlab and validated regarding experimental data. This new model will be very helpful in designing the specimens for split Hopkinson bar tests and also in the interpretation of the experimental raw data.
\end{abstract}

Index Terms - Hopkinson bar test, High strain rate, Specimen design, One-dimensional wave propagation.

\section{INTRODUCTION}

Since the pioneering works of Hopkinson and Kolsky, several improvements have been undertaken on the Hopkinson-Kolsky device. Originally designed for compression loading, this technique has been extended to test materials under tensile, shear, and bi-axial loadings. The conventional Hopkinson-bar is mainly used at the high strain rate range, i.e., between 500 and 5000/s. In addition, lowimpedance 3 bars have been proposed while testing soft materials to match the bars impedance to the specimen impedance. Namely, polymeric, or hollow aluminum Hopkinson bars have been used. The study in ref. [1] compared and reviewed the result of three important papers by B. Hopkinson, H. Kolsky and RM Davies and produced the important guidelines that help to get good result. The split Hopkinson bar test (SHPT) can be used to describe either ductile or brittle materials. In second case, the materials undergo significant inelastic deformation. This almost happens with ductile metals and polymers where significant plastic deflection occurs. The plastic deformation energy is partially or totally transformed to heat, which is either dissipated to the atmosphere or to the bars. Nevertheless, the useful test time, which is of some hundreds of microseconds in high strain rate tests, is short compared to the heat transfer or dissipation time. Hence, almost no heat is lost during the useful duration of a high strain rate test. The specimen deformation is an adiabatic [2]-[5]. The accumulation of heat induces a temperature rise in the specimen.

Several numerical works have dealt with modelling and simulation Split Hopkinson bar tests, either in compression,

Submitted on February 08, 2021

Published on March 26, 2021.

Ahmad R. Aljohani, Mechanical Engineering Department, Faculty of Engineering, King Abdulaziz University, Saudi Arabia.

(e-mail: ahmad2624@gmail.com). tension, or torsion. Gang undertook torsional finite element analysis for dynamic deflection of plastic characteristics using LS - DYNA software [6]. The strain result from simulation of input and output bar and strain-stress curves having good agreements of experimental data. There is no effect of diameter of specimen in the torsional examination and there is small effect of the specimen thickness on the experiment data with theory to measure the stress of specimen. However, the change of thickness in the shear and torsional examination having non-uniformity of strain rate, strain, and stress. In other work by using LS - DYNA software, Kariem et al. [7] exanimated to increase the number of the specimens in a test by using two specimens.

Also, the geometry and dimension of specimens have important effect on material characteristics. Prabowo et al. [8] conducted the influence of specimen geometry on the material dynamic behavior using LS - DYNA software. The research applied on three different geometry material of dumbbell shape like ASTM A370 for 6, 8, and 8 of gage length with different diameter size using Johnson - cook model and the result showing the ratio of $(\mathrm{L} / \mathrm{d}=0.75)$ having perfect strain and stress characteristics.

The type of the specimen material that is used in the Split Tensile Hopkinson bar (STHB) also have a substantial influence. Ref. [9] studied these tensile tests on a mild steel and they investigated locking influence on stain, stress, and strain rate of specimens that has initial necking. Also, the influence of pulse shapers on stainless steel alloy by using two dimensional finite element method (FEM) [10].

Most of works published in the literature have used finite element method to model split Hopkinson bar tests. In this work, we aim at developing a simplified tool with a very low computation cost. The model here will be based on the onedimensional wave theory of bars.

\section{MethodOLOGY}

The main goal of this work is to develop a simplified model for Hokinson bar tests based on analyses of wave propagation in bars. The objective is to develop a simple approach based on the one-dimensional propagation theory. Thus, it will be possible to derive theoretical solution and later build a simple MATLAB program that can be run is a short time. The split Hopkinson pressure set-up is an experimental device to test materials under impact loadings. The set-up is constituted by

Ramzi Othman, Mechanical Engineering Department, Faculty of Engineering, King Abdulaziz University, Saudi Arabia.

Khalid H. Almitani, Mechanical Engineering Department, Faculty of Engineering, King Abdulaziz University, Saudi Arabia. 
two long elastic bars: the input and output bars. The specimen is sandwiched between the two bars. The input bar is impacted by a striker bar having an impact velocity $V_{i m p}$. The impact of the striker bar generates a compressive wave in the input bar. This wave is called the incident wave, and it propagates along the input bar until it reaches the specimen. Upon arrival at the incident bar-specimen interface, part of the wave reflects back within the incident bar as a traction wave, the reflected wave. Another part is transmitted to the output bar through the specimen. It is called the transmitted wave.

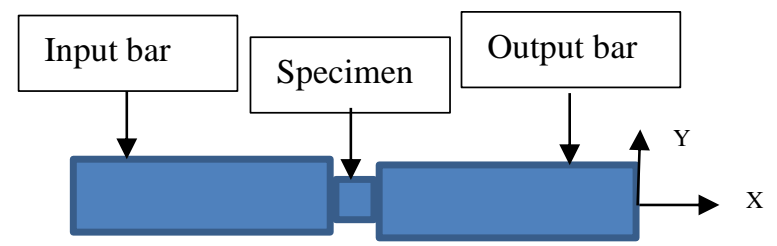

Fig. 1. Model of the Hopkinson bar setup.

Let $\varepsilon_{\text {inc }}, \varepsilon_{\text {ref }}$ and $\varepsilon_{\text {tra }}$ are the strains due to the incident wave, the reflected wave, and the transmitted wave, respectively. The incident wave strain is controlled by the velocity of the striker bar.

$$
\varepsilon_{i n c}=\frac{V_{i m p}}{2 C_{b}}
$$

where $C_{b}$ is the wave velocity in the bars. The stress in the input bar is the sum of the strains due to the incident and reflected waves:

$$
\sigma_{i n p}=E_{b}\left(\varepsilon_{i n c}+\varepsilon_{r e f}\right)
$$

where $E_{b}$ is the Young's modulus of the two bars, which are assumed made of the same material. The stress at the output bar is only due to the transmitted wave. Thus, it is equal to:

$$
\sigma_{\text {out }}=E_{b} \varepsilon_{\text {tra }}
$$

The force applied to the specimen from the input bar side is equal to:

$$
F_{\text {inp }}=E_{b} A_{b}\left(\varepsilon_{\text {inc }}+\varepsilon_{\text {ref }}\right),
$$

where $A_{b}$ is the cross-sectional area of the bars, also assumed having the same cross-sectional area.

Similarly, the force applied to the specimen from the output bar side is equal to:

$$
F_{\text {out }}=E_{b} A_{b} \varepsilon_{\text {tra }}
$$

One of the fundamental assumptions of the split Hopkinson pressure bar set-up is that the specimen is under equilibrium during the test. Thus, the input and output forces should be equal:

$$
F_{\text {out }}=F_{\text {inp }}
$$

Thus, the stress in the specimen is equal to:

$$
\sigma_{s p}=\frac{F_{\text {inp }}}{A_{s p}}=\frac{F_{\text {out }}}{A_{s p}}
$$

where $A_{s p}$ is the cross-sectional area of the specimen, which should be lower than the cross-sectional area of the bars. Hence, the stress within the specimen is higher than the stress within the bars. In terms of waves, the stress in the specimen is written as [1], [11]:

$$
\sigma_{s p}=\frac{E_{b} A_{b}\left(\varepsilon_{i n c}+\varepsilon_{r e f}\right)}{A_{s p}}=\frac{E_{b} A_{b} \varepsilon_{t r a}}{A_{s p}}
$$

In a split Hopkinson pressure bar test, the three waves $\varepsilon_{\text {inc }}$, $\varepsilon_{\text {ref }}$ and $\varepsilon_{\text {tra }}$ are measured separately. Thus, it is possible to determine the stress within the specimen.

It is also possible to calculate the strain and strain-rate in the specimen. The velocity at the input bar-specimen interface is given by:

$$
V_{\text {inp }}=-C_{b}\left(\varepsilon_{\text {inc }}-\varepsilon_{\text {ref }}\right)
$$

Similarly, the velocity at the output bar-specimen interface is given by:

$$
V_{\text {out }}=-C_{b} \varepsilon_{\text {tra }}
$$

The strain-rate in the specimen is equal to:

$$
\dot{\varepsilon}_{s p}=\frac{\Delta V}{l_{s p}}=\frac{V_{\text {out }}-V_{\text {in }}}{l_{s p}}
$$

Substituting (9) and (10) in (11) gives:

$$
\dot{\varepsilon}_{s p}=\frac{\Delta V}{l_{s p}}=\frac{-C_{b} \varepsilon_{t r a}+C_{b}\left(\varepsilon_{i n c}-\varepsilon_{r e f}\right)}{l_{s p}}
$$

Considering (4), (5), and (6), we have:

$$
\varepsilon_{\text {tra }}=\varepsilon_{\text {inc }}+\varepsilon_{\text {ref }}
$$

Substituting (13) into (12), the strain-rate in the specimen is simply expressed as [1], [11]:

$$
\dot{\varepsilon}_{s p}=\frac{-2 C_{b} \varepsilon_{r e f}}{l_{s p}}
$$

Equation (14) give an expression of the specimen strainrate in terms of the reflected wave. Thus, once the strain of the reflected wave is measured, it is possible to determine the specimen strain-rate. Later (14) is integrated to have the specimen strain [1], [11]:

$$
\varepsilon_{s p}=\int \frac{-2 C_{b} \varepsilon_{r e f}}{l_{s p}} d t
$$

\section{A. Numerical Model}

In this numerical model, we would like to develop a tool which capable of simulating the split Hopkinson bar test. We would like to develop a tool that simulates the signals that are obtained by this type of test. Knowing the properties of the bars and the specimen the numerical tool should be able to 
simulate the three bars recorded on the bar and the strain stress and strain-rate of the specimen.

\section{B. Input}

For our work, we assume the properties of the bars are known. In split Hopkinson bar set-ups, bars are mostly made of hard steel. Their diameters range commonly from 10 to $40 \mathrm{~mm}$ and their lengths range from 1 to $3 \mathrm{~m}$. Here the bars diameter is $16 \mathrm{~mm}$. The input and output bars are $2-\mathrm{m}$ long, and the projectile bar is $0.5 \mathrm{~m}$ long. All three bars have a Young's modulus and a density of $200 \mathrm{GPa}$ and $7800 \mathrm{~kg} / \mathrm{m}^{3}$, respectively. The impact velocity of the projectile bar ranges commonly between 5 to $20 \mathrm{~m} / \mathrm{s}$. Here we will choose the value of $15 \mathrm{~m} / \mathrm{s}$. The specimen used in split Hopkinson bars are of different materials and commonly of some $\mathrm{mm}$ in diameter and some $\mathrm{mm}$ in length. Here the specimen is assumed aluminum. The specimen length and diameter are considered $5 \mathrm{~mm}$ each. The specimen Young's modulus is considered $70 \mathrm{GPa}$ and its density is $2800 \mathrm{~kg} / \mathrm{m}^{3}$. The three bars should undergo only elastic deformation. However, the specimen can be deformed beyond its elastic limit. Thus, a simple linear hardening equation is assumed for the plastic deformation of the specimen:

$$
\epsilon_{s p}=\mid \begin{gathered}
\epsilon_{s p}^{\text {elas }} \text { if } \sigma_{s p} \leq \sigma_{y} \\
\epsilon_{s p}^{\text {elas }}+\epsilon_{s p}^{\text {plas }} \text { if } \sigma_{s p}>\sigma_{y}
\end{gathered}
$$

where $\epsilon_{s p}^{\text {elas }}$ and $\epsilon_{s p}^{\text {plas }}$ are the elastic and plastic strains of the specimen, and $\sigma_{y}$ is the specimen's yield stress.

The elastic strain is given by:

$$
\epsilon_{s p}^{\text {elas }}=\frac{\sigma_{s p}}{E_{s p}}
$$

where $E_{s p}$ is the Young's modulus of the specimen.

The stress during plastic deformation is given by:

$$
\sigma_{s p}=\sigma_{y}+B \epsilon_{s p}^{p l a s}
$$

where $B$ is a material constant.

TABLE I: MECHANICAL PROPERTIES OF ALUMINUM ALloy (6061-T6)

\begin{tabular}{cccc}
\hline \hline Material & $\begin{array}{c}\boldsymbol{\sigma}_{\boldsymbol{y}} \\
(\mathrm{Pa})\end{array}$ & $\begin{array}{c}\boldsymbol{B} \\
(\mathrm{Pa})\end{array}$ & $\begin{array}{c}\boldsymbol{E}_{\boldsymbol{s} \boldsymbol{p}} \\
(\mathrm{Pa})\end{array}$ \\
\hline $\begin{array}{c}\text { aluminum alloy } \\
(6061-\mathrm{T} 6)\end{array}$ & $650 \mathrm{E}+6$ & $1200 \mathrm{E}+6$ & $70 \mathrm{E}+9$ \\
\hline \hline
\end{tabular}

\section{Output}

The numerical model should calculate:

$\varepsilon_{\text {inc }}:$ the strain due incident wave,

$\varepsilon_{\text {ref }}:$ the strain due to the reflected wave,

$\varepsilon_{t r a}:$ the strain due to the transmitted wave,

$\dot{\epsilon}_{s p}:$ the specimen strain-rate,

$\epsilon_{s p}:$ the specimen strain,

$\sigma_{s p}:$ the specimen stress.

\section{Iterative Procedure}

In order to solve the problem, an iterative procedure is proposed. The time is discretized in steps. The time step is $\Delta t$ and the maximum time is $T$. All equations here are coupled and to solve them, we assume the strain due to the transmitted wave does not vary significantly during a step. Thus, we can calculate the parameters at a step $n$ using the value of the strain of the transmitted wave at a step $n-1$.

In the beginning we assume that all parameters are equal to 0 . This is the initiation step.

Thus, for the first step $\varepsilon_{i n c}, \varepsilon_{r e f}, \varepsilon_{t r a}, \dot{\epsilon}_{s p}, \epsilon_{s p}$, and $\sigma_{s p}$ are all equal to 0 . This is true just before impact.

In a general step $n$ we start by calculating the incident wave as given by Eq. (1). Thus,

$$
\varepsilon_{i n c}(n)=\frac{-\mathrm{V}_{i m p}}{2 * \mathrm{C}_{b}}
$$

Equation (13) gives a relation between the three waves $\varepsilon_{\text {inc }}, \varepsilon_{\text {ref }}$, and $\varepsilon_{\text {tra }} . \varepsilon_{\text {inc }}$ is determined by (19). In order to have separately $\varepsilon_{r e f}$, and $\varepsilon_{\text {tra }}$, we need to make an assumption either on $\varepsilon_{\text {ref }}$, or $\varepsilon_{\text {tra }}$. Here, we have assumed that $\varepsilon_{\text {tra }}$ does not vary a lot in a step. Thus, we can calculate $\varepsilon_{r e f}$ in a step $n$ using the value of $\varepsilon_{\text {tra }}$ in a step before. Mainly,

$$
\varepsilon_{r e f}(n)=\varepsilon_{t r a}(n-1)-\varepsilon_{i n c}(n)
$$

Once the reflected wave is determined, it is possible to calculate the strain-rate in the specimen using (14):

$$
\dot{\varepsilon}_{s p}(n)=\frac{-2 C_{b} \varepsilon_{r e f}(n)}{l_{s p}}
$$

By integrating the strain-rate, it is possible to calculate the specimen strain:

$$
\varepsilon_{s p}(n)=\varepsilon_{s p}(n-1)-\dot{\varepsilon}_{s p}(n) * \Delta t
$$

In order to calculate the specimen stress, we assume first an elastic behavior of the specimen. Therefore,

$$
\sigma_{s p}(n)=E_{s p} \epsilon_{s p}(n)
$$

However, we need to check the validity of the elastic assumption. Thus, the obtained specimen stress is compared to the yield stress of the specimen.

If the specimen stress is lower than the yield stress, $\left(\sigma_{s p}(n) \leq \sigma_{y}\right)$ then the assumption is true, and the specimen stress calculated using (23) is correct and maintained. In this case, we need just to update the strain due to the transmitted wave using (8):

$$
\varepsilon_{t r a}(n)=\frac{A_{s p}}{E_{b} A_{b}} \sigma_{s p}(n)
$$

Also, we consider that the plastic deformation is absent:

$$
\epsilon_{s p}^{\text {plas }}(n)=0
$$

With this equation a step $n$ is closed if the deformation of the specimen is elastic. We then move to the next step using (19). 
If the stress obtained by (23) is higher than the yield stress $\left(\sigma_{s p}(n)>\sigma_{y}\right)$, then the specimen undergoes some plastic deformation. The result of (23) is not correct and thus ignored. Thus, the specimen stress is calculated using (18). However, this equation involves the plastic deformation which is not calculated yet. We then assumed that the plastic deformation does not vary significantly within a step. Consequently, we calculate the stress using the plastic deformation in the previous step:

$$
\sigma_{s p}(n)=\sigma_{y}+B \epsilon_{s p}^{p l a s}(n-1)
$$

Once the specimen stress is determined, it is possible to calculate the elastic strain using (17):

$$
\epsilon_{s p}^{e l a s}(n)=\frac{\sigma_{s p}(n)}{E_{s p}}
$$

Subsequently, we calculate the plastic strain using (16):

$$
\epsilon_{s p}^{\text {plas }}(n)=\epsilon_{s p}(n)-\epsilon_{s p}^{\text {elas }}(n)
$$

We can finally calculate the strain due to the transmitted wave using (24) and the step is closed.

\section{RESULTS AND DISCUSSION}

In this section, we are going to present the results obtained by the analytical one-dimensional model of the split Hopkinson pressure bar. First, we are going to present a validation of the model. Subsequently, several parametric study will be presented.

\section{A. Validation}

The one-dimensional numerical model is validated by comparison to experimental data. Fig. 2 shows a comparison between reflected and transmitted waves that are obtained either by numerical model or by a split Hopkinson bar test. The specimen in both cases is aluminum for which data is presented in Table I. In this case study, the impact velocity of the striker bar is $15 \mathrm{~m} / \mathrm{s}$. The striker bar is also $0.5 \mathrm{~m}$ long. The bar diameter is $16 \mathrm{~mm}$. The specimen is $5 \mathrm{~mm}$ in length and $5 \mathrm{~mm}$ in diameter. The bars are made of steel of Young's modulus $200 \mathrm{GPa}$ and density $7800 \mathrm{~kg} / \mathrm{m}^{3}$.

The numerical model predicts well the reflected and transmitted waves, mainly, in the post-yield behavior. The elastic slope in the beginning of the test is higher in the numerical waves than the experimental waves because of the inertia in the beginning of the test. The numerical model assumes perfect equilibrium of the specimen. Thus, it cannot take into account the specimen inertia.

The numerical model cannot catch the oscillations that are present in the experimental waves. Indeed, these oscillations are due to wave dispersion caused by radial inertia of the bars. However, the numerical model is based on the onedimensional wave theory. Thus, it cannot include radial inertia of bars.

However, the numerical is predicting well the waves in the plastic part, which is the most important part of the test. Thus, the numerical model can predict well the strain rate, the plastic strain, and the stress in the post-yield part as shown in Fig. 3 and 4.

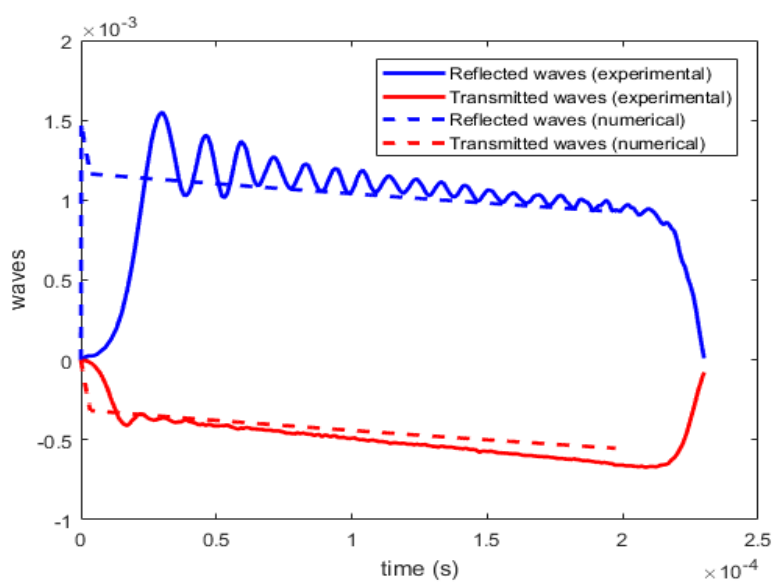

Fig. 2. Reflected and transmitted waves of SHPT.

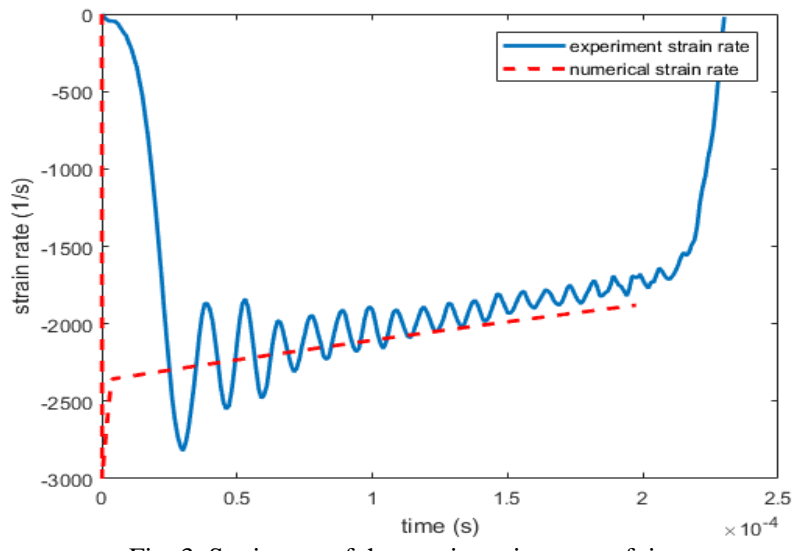

Fig. 3. Strain rate of the specimen in terms of time.

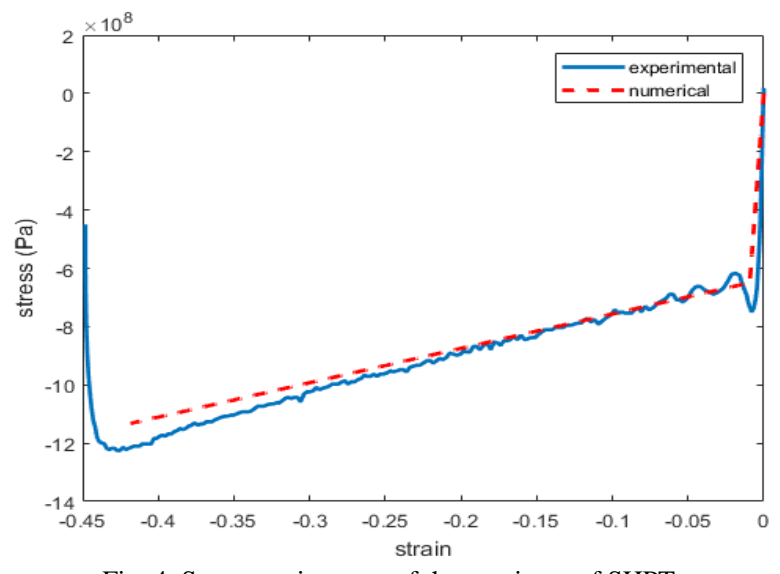

Fig. 4. Stress-strain curve of the specimen of SHPT.

\section{B. Effect of Impact Velocity}

As parametric study, we have also studied the effect of the impact velocity on the strain rate and waves signals in a split Hopkinson pressure bar test. Fig. 5 shows the strain in terms of time for a striker bar velocity ranging between 5 and $20 \mathrm{~m} / \mathrm{s}$. As the impact velocity increases the strain rate increases too. This is expected as by increasing the striker impact velocity, higher kinetic energy is delivered to the specimen for the same period of time. Thus, the specimen will be more deformed for the same time if the impact velocity is increased. Hence the strain-rate is increased. 
Fig. 6 shows transmitted waves in terms of time for several impact velocities. As the impact velocity, the strain-rate increases. For the same period of time, the strain level increases for higher impact velocities. If higher strain levels are reached it means higher stress levels are reached. Thus, transmitted waves are higher because transmitted waves are proportional to the specimen stress.

Fig. 7 shows reflected waves in terms of time for several impact velocities. Reflected waves are proportional to the specimen strain rate. If the impact velocity is higher, then the strain rate is higher. Consequently, the level of the reflected waves is higher.

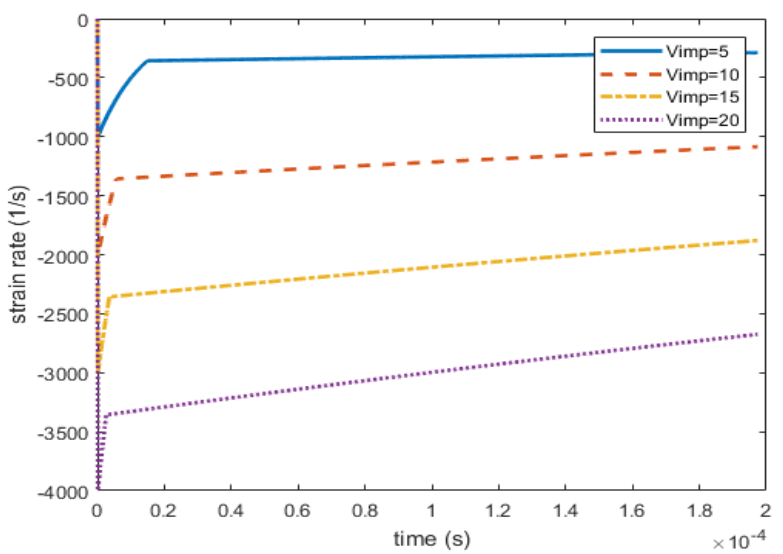

Fig. 5. Strain rate of the specimen for different impact velocities.

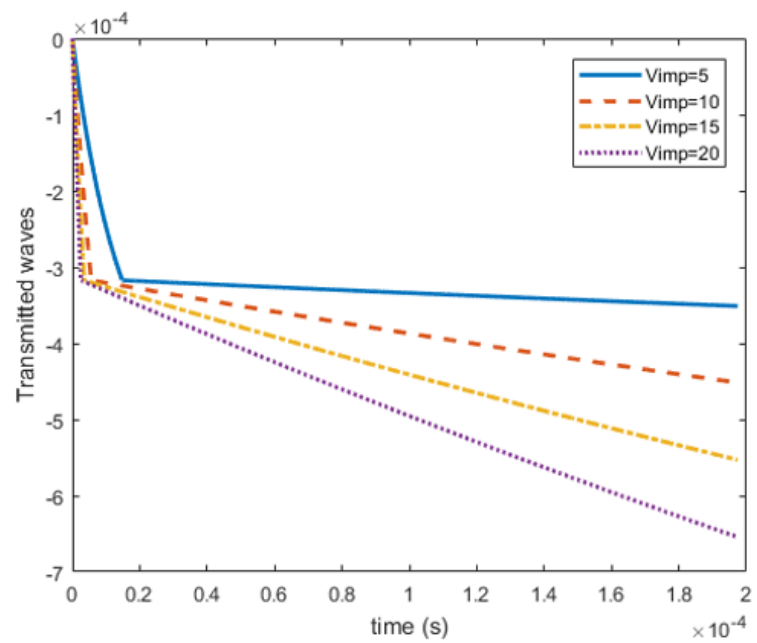

Fig 6. Transmitted waves in terms of time for different impact velocities.

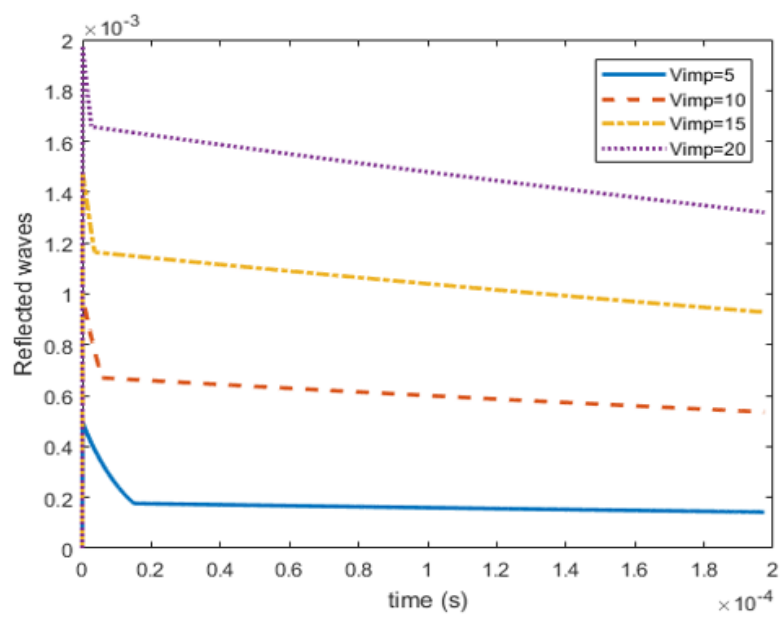

Fig 7. Reflected waves in terms of time for different impact velocities.

\section{CONCLUSION}

A one-dimensional numerical approach is developed here to predict wave signals in a split Hopkinson pressure bar test. The approach considered here is simple and with very low computation time cost. The numerical approach is implemented using MATLAB. It is validated here by comparing reflected and transmitted waves of numerical and experimental SHPB test on an aluminum alloy. The numerical predicts well the post-yield behavior. The numerical model is then used to study the effect of the striker bar impact velocity. It is showed that the specimen strain-rate increases with the impact velocity.

\section{REFERENCES}

[1] Gama, Bazle A., Sergey L. Lopatnikov, and John W. Gillespie Jr. "Hopkinson bar experimental technique: a critical review." Appl. Mech. Rev. 57, no. 4 (2004): 223-250.

[2] Chou, S. C., K. D. Robertson, and J. H. Rainey. "The effect of strain rate and heat developed during deformation on the stress-strain curve of plastics." Experimental mechanics 13.10 (1973): 422-432.

[3] Mason, J. J., A. J. Rosakis, and G. Ravichandran. "On the strain and strain rate dependence of the fraction of plastic work converted to heat: an experimental study using high speed infrared detectors and the Kolsky bar." Mechanics of Materials 17.2-3 (1994): 135-145.

[4] Noble, J. P., and J. Harding. "Temperature measurement in the tensile Hopkinson bar test." Measurement Science and Technology 5.9 (1994): 1163.

[5] Kapoor, Rajeev, and Sia Nemat-Nasser. "Determination of temperature rise during high strain rate deformation." Mechanics of materials 27.1 (1998): 1-12.

[6] Gang, Chen, et al. "Numerical Simulation on the specimen dynamic plastic deformation behaviour in the torsional split Hopkinson bar test." EPJ Web of Conferences. Vol. 183. EDP Sciences, 2018.

[7] Kariem, Muhammad Agus, John H. Beynon, and Dong Ruan. "Numerical simulation of double specimens in split Hopkinson pressure bar testing." Materials Science Forum. Vol. 654. Trans Tech Publications Ltd, 2010

[8] Prabowo, Dini A., Muhammad A. Kariem, and Leonardo Gunawan. "The effect of specimen dimension on the results of the SplitHopkinson tension bar testing." Procedia engineering173 (2017): 608 614.

[9] Mirone, G., R. Barbagallo, and F. Giudice. "Locking of the strain rate effect in Hopkinson bar testing of a mild steel." International Journal of Impact Engineering 130 (2019): 97-112.

[10] Ameri, A. A. H., et al. "An effective pulse-shaping technique for testing stainless steel alloys in a split-Hopkinson pressure bar." Journal of Dynamic Behavior of Materials 5.1 (2019): 39-50.

[11] Chen, Weinong W., and Bo Song. Split Hopkinson (Kolsky) bar: design, testing and applications. Springer Science \& Business Media, 2010 .

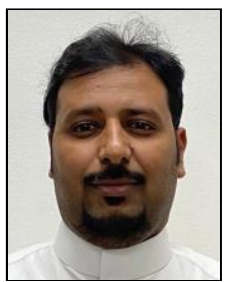

Ahmad R. Aljohani

Date of birth: 1985, Yanbu Saudi Arabia.

Education: B.S. in Mechanical Engineering, King Abdulaziz University, Jeddah, Saudi Arabia 2010.

(On going) M.S. in Mechanical Engineering, King Abdulaziz University, Jeddah, Saudi Arabia. 